\title{
Water, climate, and health
}

\author{
Hwa-Lung Y $\mathbf{u}^{1}$
}

Published online: 21 November 2016

(c) Springer-Verlag Berlin Heidelberg 2016

Water is among the cores of the sustainable environment and human health. It is evident that climate change has been imposing a variety of stresses on changing water quantity and quality across the globe. For example, the climate change has increased the temperature and perturbed the hydrological processes in terms of changing the occurrence frequency of extreme water events. These changes have been considered to be the important causes in the water quality changes, water deficiency, and the disturbance of ecological and environmental processes in recent decades. As a result, these associated changes can have impacts on human's health in various paths, such as the increases of the magnitude and frequencies of waterrelated diseases, and water-related disasters. There is an urgent need to have a better understanding of the interactions among water, climate, and health. In response to the emerging concern of this cross-disciplinary research topic, this special issue carefully selected the papers from the authors with multiple disciplinary backgrounds to address a variety of perspectives on the connections among the contexts of water, climate, and health. Among them, many papers are invited from the presentations of the European Geosciences Union (EGU) session of "Water, Climate, and Health" in the years of 2013 and 2014.

Understanding the space-time variations of water-borne diseases is an essential strategy to assess the climate change impact on the disease occurrences and spread. The basic idea is to assess how the major environmental and hydrological factors can influence the aquatic environment

Hwa-Lung Yu

hlyu@ntu.edu.tw

1 Department of Bioenvironmental Systems Engineering, National Taiwan University, Taipei 10617, Taiwan for the bacteria or pathogens, and therefore the space-time distribution of the water-borne disease incidences. Two selected papers investigated the space-time occurrences and spreads of cholera epidemics. In Bertuzzo and his colleagues' paper, a spatially-explicit stochastic susceptible-Infected-recovered (SIR) model was proposed to account for the environmental concentration of cholera and to estimate the extinction probability of cholera in Haiti which has caused more than 8500 deaths and high fatality rate in its 2010 epidemic. Augustijn and her colleagues took a micro-scale approach by presenting a spatially explicit agent-based model to assess the cholera diffusion in the 2005 epidemic in Ghana. To assess the climate change impact, McBride, Tait, and Slaney used the projected rainfalls and temperature under several selected the climate change scenarios to evaluate the potential changes in the exposure to the pathogens campylobacteriosis and cryptosporidiosis in 2040 and 2090 in New Zealand by using a SIR model.

The vector-borne diseases have also been shown its high sensitivity to the changes of water and climatic variations, because the temperature and hydrological factors can have a strong impact on the living conditions of disease vectors. Lowe and her colleagues showed that, by using both the generalized linear and additive mixed models, an additional $8 \%$ of dengue fever relative risks could result from the inter-annual variations in precipitation and temperature in the previous month in Thailand. Another statistical model was proposed in $\mathrm{Yu}$, Lee, and Chien's work to assess the time lag-magnitude interactive effects of rainfalls and temperature on dengue fever incidences in Taiwan. In their work, they identified the important thresholds of temperature and rainfalls for the prediction of dengue fever risks in space and time. Lin and her colleagues conducted a survey of dengue vectors, including Aedes 
aegypti and Aedes albopictus, in both indoors and outdoors and found that the spatial distributions of these two vectors presented distinct features in spatial clusters at different stages during the 2010 epidemic in Kaohsiung, Taiwan.

The exposure assessment in water-related health risk analysis requires not only the knowledge about the concentrations of pathogens or pollutants, but also the human activities related to the water contact that are essential in evaluating the possibility and frequency of the exposure to the disease agents. To improve the exposure assessment, Traore and his colleagues conducted a field survey at Sourou Valley in Burkina Faso to evaluate an empirical relationship between the temperature increases and the frequency changes in children's exposure to the water in terms of swimming and bathing, in order to assess the potential schistosomiasis transmission risk under the climate change.

To better characterize the hydrological processes is important to the health risk modeling. This issue selected two advanced approaches that provide a closer look on hydrological conditions from the collection and analysis of the space-time observations. Muchebve and his colleagues conducted a wavelet coherence method to account for the nonlinear relationship between a variety of hydrological and meteorological factors and the seawater intrusion flux to groundwater, which can worsen the groundwater quality and therefore have impacts on human and ecological health conditions. Kim and his colleagues obtained space-time distributions of soil moisture by assimilating the remote sensing and in situ observations. In many applications, the understanding of soil moisture is essential to the further climatic, hydrological and health risk analyses.

I would like to thank the authors for their outstanding research contributions and, in particular, Prof. George Christakos (SERRA Editor-in-Chief) for his encouragement and support in preparing this special SERRA Issue. 